\title{
A randomized, controlled trial to assess short-term black pepper consumption on 24-hour energy expenditure and substrate utilization
}

\author{
Annalouise O'Connor ${ }^{1}$, Karen D. Corbin, ${ }^{1}$ David C. Nieman ${ }^{2}$, Andrew G. Swick ${ }^{1 *}$ \\ ${ }^{1}$ UNC Nutrition Research Institute, University of North Carolina Chapel Hill, North Carolina \\ Research Campus, Kannapolis, North Carolina, USA; ${ }^{2}$ Human Performance Laboratory, \\ Appalachian State University, North Carolina Research Campus, Kannapolis North Carolina, \\ USA
}

*Corresponding author: Andrew G. Swick, ${ }^{1}$ UNC Nutrition Research Institute, University of North Carolina Chapel Hill, North Carolina Research Campus, Kannapolis, North Carolina, USA

Submission date: August 5, 2013; Acceptance date: October 12, 2013; Publication date: October 15,2013

\begin{abstract}
Background: Thermogenic ingredients may play a role in weight management. In vitro and rodent work suggests that components of black pepper may impact energy expenditure, and in humans, other TPRV1 agonists e.g. capsaicin, augment EE.
\end{abstract}

Objectives: To determine the impact of $\mathrm{BP}$ on 24-hour $\mathrm{EE}$, respiratory quotient, and biochemical markers of metabolism and satiety, a randomized, controlled, cross-over study of black pepper $(0.5 \mathrm{mg} / \mathrm{meal})$ versus no pepper control was conducted in post-menopausal women. Subjects spent two 24-hour periods in a whole room indirect calorimeter.

Results: Post-meal glucose, insulin, gut peptides and catecholamines were measured. Energy expenditure, respiratory quotient, or biochemical markers assessed did not differ significantly between the black pepper and no pepper control study days.

Conclusions: Our findings do not support a role for black pepper in modulating energy expenditure in overweight postmenopausal women. Future work targeting alternative populations, administering black pepper in the fasted state, or in combination with other spices, may reveal the thermogenic effect of this spice.

Trial registration: This trial was registered at clinicaltrials.gov (NCT01729143).

Key words: Black pepper, piperine, energy expenditure, metabolic chamber 


\section{BACKGROUND}

Rising obesity rates are alarming considering the negative impact on morbidity, mortality and the economics of health care. Alongside lifestyle interventions, functional foods are gaining support as weight management tools.

Several functional ingredients have been demonstrated to increase energy expenditure (EE) [1]. Of the bioactive spices, the thermogenic and satiating properties of capsaicin, the major pungent principle in hot red pepper (RP), have been studied in some detail [2-3]. Capsaicinmediated activation of the human vanilloid receptor 1 (TRPV1) leading to increased catecholamine secretion and sympathetic nervous system (SNS) activation, is the suggested thermogenic-enhancing mechanism [1]. However, issues surrounding compliance with longerterm capsaicin and hot RP intake in Western populations [4] raises questions about the ingredient's realistic role in weight management.

Piperine (1-peperoylpiperidine), the alkaloid contributing to the pungency of black pepper (BP), is also a TRPV1 agonist, and although less potent, showed greater efficacy at TRPV1 than capsaicin [5]. Evidence of increased catecholamine secretion in rats fed piperine [6], represents a plausible mechanism by which piperine may increase EE. Piperine has also been shown to influence gastric emptying and transit time in rodents [7-8] and piperine suppressed adiposity in mice without changing caloric intake [9].

Based on this positive cell and animal data, as well as the thermogenic capacity of other TRPV1 agonists, our objective was to test the hypothesis that dietary supplementation with BP would increase 24-hour EE. The study was limited to overweight post-menopausal women, an at risk group in need of targeted dietary guidance.

\section{METHODS}

Study Design: This was a randomized, controlled, cross-over trial of BP (1.5g/day) versus no pepper control (NPC). Based on a US Department of Agriculture (USDA)-led analysis of BPs, this corresponds to $68.55 \mathrm{mg} /$ day of piperine (45.7mg piperine/g BP) [10]. The study consisted of two 24-hour periods in the metabolic chamber at the University of North Carolina Chapel Hill Nutrition Research Institute (UNC NRI), each one week apart (Figure 1). Random allocation sequence was generated using randomization.com (randomly permuted blocks). Clinical and research staff randomized and enrolled subjects. Primary outcome measures: differences in 24hour EE and substrate utilization (indicated by respiratory quotient (RQ)). Secondary outcome measures: changes in postprandial (30 minutes after lunch) gut peptides (gastric inhibitory polypeptide (GIP), glucagon-like peptide 1 (GLP-1), peptide tyrosine tyrosine (PYY), pancreatic polypeptide (PP), ghrelin) and other relevant biomarkers (insulin, leptin, amylin, adrenaline, noradrenaline, dopamine). Subjects were recruited February-June 2011. The study was conducted between April and July 2011.

To determine the effects of BP consumed as part of a meal, subjects consumed $60.8 \mathrm{~g}$ of low-sodium V8® vegetable juice (Campbell Soup Company, Camden, NJ, USA) with or without $0.5 \mathrm{~g}$ BP (McCormick®, Sparks, MD, USA) at each of the 3 study day meals. To ensure full BP delivery, subjects were asked to rinse and drink any BP from the sides of the cup. Due to the method of BP administration, subjects were not blinded to the treatment. During both study days, subjects were fed in energy balance, meaning that energy intake was matches with EE. 
Pre-study taste test: To assess the palatability of several culinary doses of BP $(0 . \mathrm{g}, 0.7 \mathrm{~g}$, $1.0 \mathrm{~g} / \mathrm{serving}$ ), a taste panel including 8 volunteers was developed. On a 5-point Likert scale, subjects indicated their feelings about a particular dose acceptability (1:Love it to 5:Yuck) and ability to consume a dose 3 times/day (1:Definitely to 5: No way). Based on these responses, a 3 times/day dose of $0.5 \mathrm{~g}$ was deemed most acceptable. A burning sensation in the mouth was reported with higher doses. The daily average intake of BP in the population from which this sample was drawn is $0.3 \mathrm{~g} /$ day [10-12]. During the chamber study screening process, all subjects consumed a one-meal test dose of $0.5 \mathrm{~g}$, to ensure acceptability.

Study participants: This study was conducted according to guidelines laid down in the Declaration of Helsinki (as revised in 1983) and was registered retrospectively at clinicaltrials.gov (ID NCT01729143). All procedures were approved by the Institutional Review Boards (IRBs) of Appalachian State University and UNC Chapel Hill. Written informed consent was obtained from all subjects. Subjects were recruited from the local area. Inclusion criteria: female; 50-70 years; healthy; exercises <150 minutes/week; no known sensitivity/ allergy to BP; no history of drug/alcohol abuse; no herbal supplements or medications known to influence EE (e.g. capsaicin, green tea catechins, $\beta$-blockers), or which may influence the study protocol (e.g. diabetic on insulin). Post-menopausal status was ascertained by self-report (absence of menses for $>12$ months). Subjects were excluded if they did not meet inclusion criteria, or had an abnormal thyroid profile (normal reference ranges: T3, 90-178ng/dL; T4, 6.1-12.2mcg/dL; thyroid stimulating hormone (TSH), 0.3-3.0uIU/L).

Subjects attended the UNC NRI, for screening. Body composition (fat mass and fat free mass (FFM)) was determined via dual energy x-ray absorptiometry (DXA) (GE Lunar iDXA; Milwaukee, WI). Resting Metabolic Rate (RMR) was calculated using a FFM-based equation [418 + (20.3FFM)] [12]. Estimated RMR was used to calculate energy intake for the study days (RMR X physical activity level (PAL) of 1.3), and adjusted using measured data (details below). This prediction resulted in estimated kcal requirements in line with measured EE in this population ( $\mathrm{p}<0.05$ for estimated compared with measured data). Actual food consumption was further adjusted during the study day to tightly match energy intake with measured EE (see details below).

Calorimetry: Indirect calorimetry was conducted using the metabolic chamber at the UNC NRI, which was modelled on chambers at the National Institute of Diabetes and Digestive and Kidney Diseases, Bethesda, MD [13]. It is an open-circuit, pull-type, whole-room indirect calorimeter built with walk-in cooler panels [14]. This chamber and the reproducibility of the data generated has been previously described [15]. EE was calculated using an abbreviated Weir's formula $\left(\mathrm{VO}_{2} \mathrm{X} 3.941\right)+\left(\mathrm{VCO}_{2} \mathrm{X} 1.106\right)$, where $\mathrm{VO}_{2}$ is the volume of oxygen consumed (L/minute) and $\mathrm{VCO}_{2}$ is the volume of carbon dioxide released (L/minute). RQ was calculated as $\mathrm{VCO}_{2} / \mathrm{VO}_{2}$. A passive infrared motion sensor measured spontaneous physical activity each minute. For calculation of RMR, EE was plotted against the activity motion sensor output (each averaged over 30 minutes), and the intercept of the linear regression taken as EE in the inactive state (RMR). This value for RMR was extrapolated to 24 hours (by multiplication by 1440 
minutes). Sleeping metabolic rate (SMR) was defined as the lowest mean EE measured over 3 consecutive hours between 0.00 and 6.30 hours and was extrapolated to 24 hours. Diet induced thermogenesis (DIT) was calculated as RMR-sleeping metabolic rate (SMR). Activity induced EE (AEE) was calculated as 24hour EE-RMR [16].

Study day protocol: Subjects reported to the unit following an overnight fast. Subjects were instructed to avoid exercise the day before, and to not consume BP, RP, alcohol or caffeine for two days prior to each visit. Subjects were weighed in light clothing without shoes. At 08.00 hours, subjects were sealed in the chamber and were requested to remain seated or reclined, but awake throughout the day. Subjects stood and stretched for 2-minutes each hour and performed necessary daily activities (e,g. using restroom) during these intervals where possible. Subjects completed an hourly activity log on visit 1 and mimicked this log on visit 2. Breakfast (at 09.00 hours), lunch (at 13.30 hours), and dinner (at 17.00 hours), were served through an air-lock passage. The V8® with or without BP was served alongside each meal (consumed within 30 minutes). At 14.00 hours blood was sampled through an iris port. Subjects were requested to prepare for bed at 22.00 hours. At 22.30 hours, lights were turned off and subjects were asked to lie down even if not sleeping. Subjects were woken at 06.30. At 07.15 hours, subjects exited the chamber.

Metabolic Diets: Diet provided 30\% kcal fat, 55\% kcal carbohydrates and 15\% kcal protein. Menus were designed by the UNC School of Medicine Clinical Translational Research Centre using ProNutra (VioCare, Princeton, NJ). The same foods (with or without BP) were served to all subjects at both chamber visits. A baseline menu for each subject was prepared based on calculated RMR X 1.3, reflecting the sedentary nature of the study day. To ensure energy balance, this baseline menu was modified based on actual measured EE: using data generated at 3 hours (includes breakfast) and 7 hours (includes breakfast and lunch), food consumption at subsequent meals was adjusted. Adjustments were consistent between study days.

Biochemical Analysis: Insulin, leptin, c-peptide, GIP, GLP-1, PYY, PP, amylin and ghrelin were analyzed using bead-based multiplex assays (Millipore, Billerica, MA) on a Luminex $100^{\mathrm{TM}}$ (Luminex, Austin, TX). Catecholamines (epinephrine, norepinephrine, dopamine) were measured by HPLC with electro-chemical detection (HPLC-EC). TSH was measured by twosite immune-enzymatic (sandwich) assay. T3 and T4 were measured by competitive binding immune-enzymatic assay. Thyroid hormone tests were run on a Beckman Unicel DXI 800 Immunoassay System.

Analysis of plasma piperine: Blood samples were collected from subjects 30 minutes after lunch (two test meals consumed). Piperine and its fragment were detected using ultraperformance liquid chromatography quadruple time-of-flight mass spectrometry (UPLCQTOFMS, Waters Corp., Milford, MS). Each plasma sample was mixed with internal standard $(50 \mu \mathrm{g} / \mathrm{ml}$ chlorophenylalanine in water). Diluted samples were extracted with organic solvent (methanol:acetonitrile=5:3, v/v). A UPLC-QTOFMS system was operated in both electrospray ionization (ESI) positive and negative modes. For ESI+ mode, the mobile phases were $0.1 \%$ 
formic acid in water and $0.1 \%$ formic acid in acetonitrile. For ESI mode, the mobile phases were acetonitrile and $10 \mathrm{mM}$ ammonium fluoride in water. Raw data files were processed using the MarkerLynx XS application manager (Waters Corp., Milford, MA). To evaluate the analytical variation for this data set, the initial data quality was assessed using pooled QC samples. For the UPLC-QTOFMS data signals detected in $>80 \%$ of all the pooled QC samples with CV $\leq 30 \%$ were included. After QC review, the UPLC-QTOFMS data were normalized to the internal standard. Metabolite annotation was performed by comparing detected signal from the study samples to those of reference standards for piperine (Sigma Aldrich).

Statistical Analysis: Using data generated previously in this chamber (ref. 16), a sample size calculation with $80 \%$ power revealed that 16 subjects were required to detect a $0.21 \mathrm{MJ}$ difference in 24-hr EE between the BP and NPC study days. Data are expressed as mean \pm SD unless otherwise stated. Statistical analyses were conducted in Prism (GraphPad Software, San Diego, CA). Data was checked for Gaussian distribution and transformed where necessary to coax normality. Differences between BP and NPC study days were assessed by paired t-tests, or the non-parametric alternative (Wilcoxon Signed Rank Test) if required. $p<0.05$ was considered statistically significant.

\section{RESULTS}

\section{Description of the study cohort}

27 women were recruited. Following screening, 26 subjects were deemed eligible. 20 subjects commenced the study, of which 18 completed all study days. One subject was excluded due to poor compliance, leaving 17 subjects in the final analysis (Figure 1). No adverse effects of the BP were reported. Clinical characteristics of the study population are detailed in Table 1. No significant differences were identified between the group randomized to complete the BP study day first $(n=7)$ and those who first completed the NPC study day (data not shown).

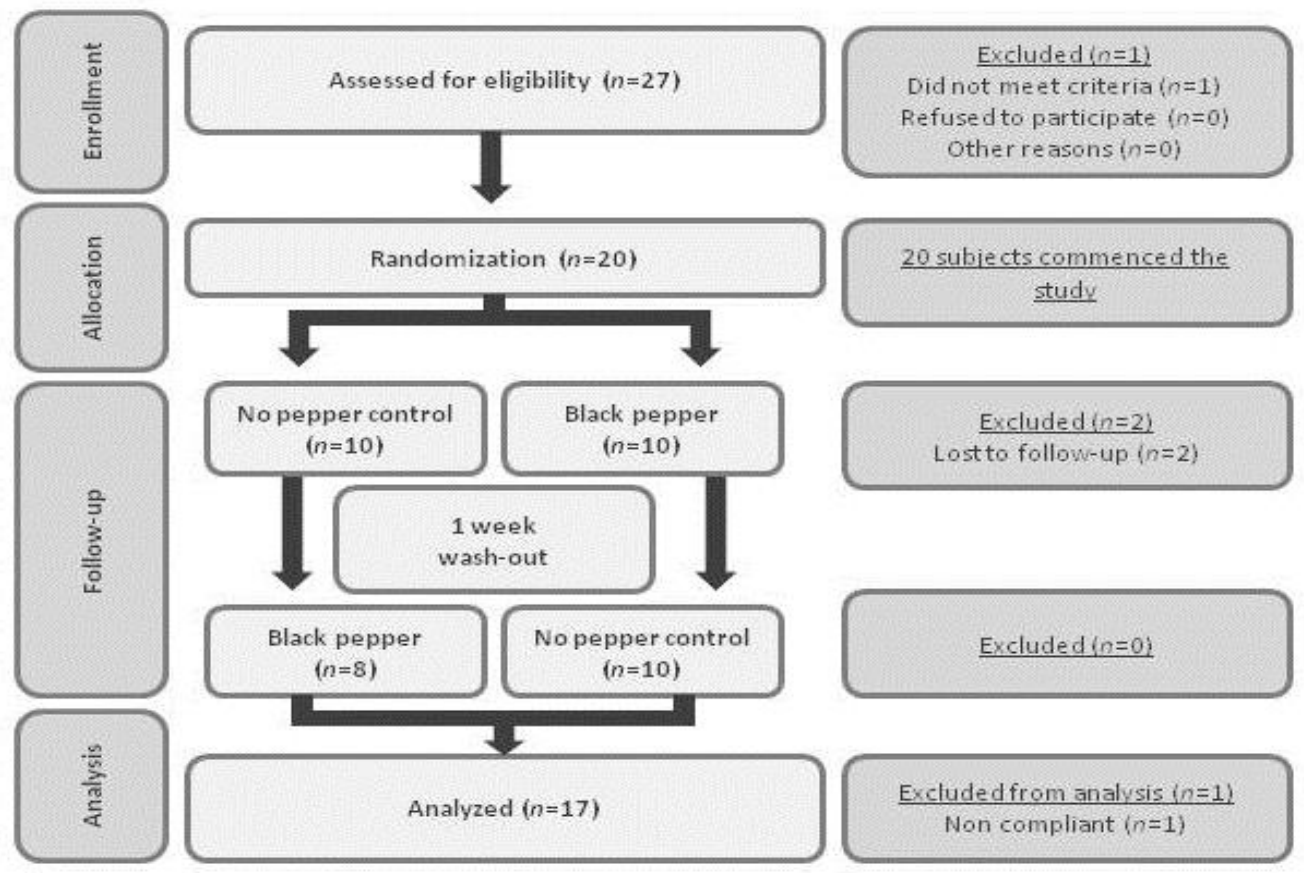

Figure 1: Flow diagram of study participants and study design 
Table 1. Clinical characteristics of post-menopausal women $(n=17)$ who completed both the black pepper and no pepper control study days ${ }^{1}$

\begin{tabular}{|c|c|c|c|}
\hline & Mean & SD & Range \\
\hline Age (years) & 60.4 & 5.2 & $52-69$ \\
\hline BMI $\left(\mathrm{kg} / \mathrm{m}^{2}\right)$ & 30.8 & 5.2 & $24.3-45.3$ \\
\hline $\operatorname{FFM}(\%)^{1}$ & 47.6 & 3.8 & $46.2-62.6$ \\
\hline Body Fat $(\%)^{1}$ & 52.4 & 3.8 & $37.4-53.8$ \\
\hline T3 (ng/dL) ${ }^{2}$ & 122.0 & 19.1 & $96-160$ \\
\hline T4 (mcg/dL) ${ }^{3}$ & 7.11 & 2.0 & $5.6-9.6$ \\
\hline TSH $(\mathrm{uIU} / \mathrm{mL})^{4}$ & 1.8 & 0.9 & $0.9-3.0$ \\
\hline \multirow[t]{2}{*}{ Race/ethnicity } & White & & 17 \\
\hline & Other & & 0 \\
\hline
\end{tabular}

${ }^{1}$ Data given as mean and standard deviation

2 as assessed by DXA

${ }^{3}$ Normal laboratory reference range: $90-178 \mathrm{ng} / \mathrm{dL}$

${ }^{4}$ Normal laboratory reference range: $6.1-12.2 \mathrm{mcg} / \mathrm{dL}$

${ }^{5}$ Normal laboratory reference range: $0.3-3.000 \mathrm{uIU} / \mathrm{mL}$

FFM, fat free mass; DXA, dual X-ray absorptiometry; TSH, thyroid stimulating hormone

\section{BP consumption increases circulating piperine}

Piperine $(m / z=286.1, \mathrm{RT}=4.38$ minutes $)$ and its fragment $(m / z=201.1)$ were identified in all samples. The average peak intensity of piperine $(389 \pm 209$ versus $1570 \pm 544)$ and its fragment (72 \pm 35 versus $286 \pm 102)$ were significantly increased $(P<0.0001$ for both) following BP consumption versus NPC.

\section{BP does not alter EE or $R Q$ in overweight, post-menopausal women}

No differences were observed in 24-hour EE or components of EE, namely RMR, SMR, DIT, or AEE between the NPC and the BP treatments (Table 2). No significant between-treatment differences were observed in 24-hour RQ or post-prandial AUC for RQ 4 hours after breakfast, lunch, or dinner (Table 3). Activity (\%) was comparable between the BP and NPC study days $(7.49 \pm 3.85$ versus $8.28 \pm 3.73 ; P=0.14)$.

Table 2. 24-hour EE and components of EE in post-menopausal women $(n=17)$ who completed the black pepper and no pepper control study days ${ }^{1}$

\begin{tabular}{|c|c|c|c|c|c|}
\hline & \multicolumn{2}{|c|}{ No pepper control } & \multicolumn{3}{|c|}{ Black Pepper } \\
\hline & $\mathrm{n}=17$ & & $\mathrm{n}=17$ & & \\
\hline & Mean & SD & Mean & & SD \\
\hline 24-hour EE (MJ/day) & 7.4 & \pm 0.9 & 7.4 & \pm & $0.9^{\mathrm{NS}}$ \\
\hline RMR (MJ/day) & 6.5 & \pm 0.8 & 6.5 & \pm & $0.8^{\mathrm{NS}}$ \\
\hline SMR (MJ/day) & 5.5 & \pm 0.7 & 5.5 & \pm & $0.7^{\mathrm{NS}}$ \\
\hline DIT (MJ/day) & 0.9 & \pm 0.5 & 0.9 & \pm & $0.5^{\mathrm{NS}}$ \\
\hline AEE (MJ/day) & 0.9 & \pm 0.4 & 0.9 & \pm & $0.4^{\mathrm{NS}}$ \\
\hline
\end{tabular}

${ }^{1}$ Data displayed as mean \pm SD. ${ }^{\text {NS }}$ indicates not significantly different from no pepper control study day as assessed by paired t-tests on normally distributed data. $P<0.05$ considered statistically significant. EE, energy expenditure; RMR, resting metabolic rate; SMR, sleeping metabolic rate; DIT, diet induced thermogenesis; AEE, activity induced energy expenditure; $\mathrm{MJ}$, mega joule $(1 \mathrm{MJ}=239 \mathrm{kcal})$ 
Table 3. 24-hour and post prandial RQ in post-menopausal women $(n=17)$ who completed the black pepper and no pepper control study days ${ }^{1}$

\begin{tabular}{|c|c|c|c|c|c|c|}
\hline & \multicolumn{3}{|c|}{ No pepper control } & \multicolumn{3}{|c|}{ Black Pepper } \\
\hline & $\mathrm{n}=17$ & & & $\mathrm{n}=17$ & & \\
\hline & Mean & & SD & Mean & & SD \\
\hline 24-hour RQ & 0.89 & \pm & 0.021 & 0.89 & \pm & $0.019^{\mathrm{NS}}$ \\
\hline Post B AUC RQ & 0.091 & \pm & 0.052 & 0.094 & \pm & $0.047^{\mathrm{NS}}$ \\
\hline Post L AUC RQ & 0.092 & \pm & 0.036 & 0.085 & \pm & $\mathbf{0 . 0 3 3}^{\mathrm{NS}}$ \\
\hline Post D AUC RQ & 0.142 & \pm & 0.090 & 0.144 & \pm & $0.070^{N S}$ \\
\hline
\end{tabular}

${ }^{1}$ Data displayed as mean \pm SD. ${ }^{\text {NS }}$ indicates not significantly different from no pepper control study day as assessed by paired t-tests on normally distributed data. $P<0.05$ considered statistically significant.

$\mathrm{RQ}$, respiratory quotient; B, breakfast; L, lunch; D, dinner; AUC, area under the curve

AUC calculated using RQ data collected during the 4 hours following B, L and D were served.

BP does not influence post-prandial biomarkers of satiety or metabolism in overweight postmenopausal women

Postprandial concentrations of gut peptides (GLP-1, PYY, GIP, ghrelin) were comparable between BP and NPC treatments (Table 4). Additionally, insulin, c-peptide, leptin, adrenaline, noradrenaline and dopamine did not differ between treatments (Table 4).

Table 4. Plasma biomarkers 30 minutes after ingestion of a mixed meal containing black pepper $(0.5 \mathrm{~g})$ compared with no pepper control in overweight post-menopausal women $(n=17)^{1}$

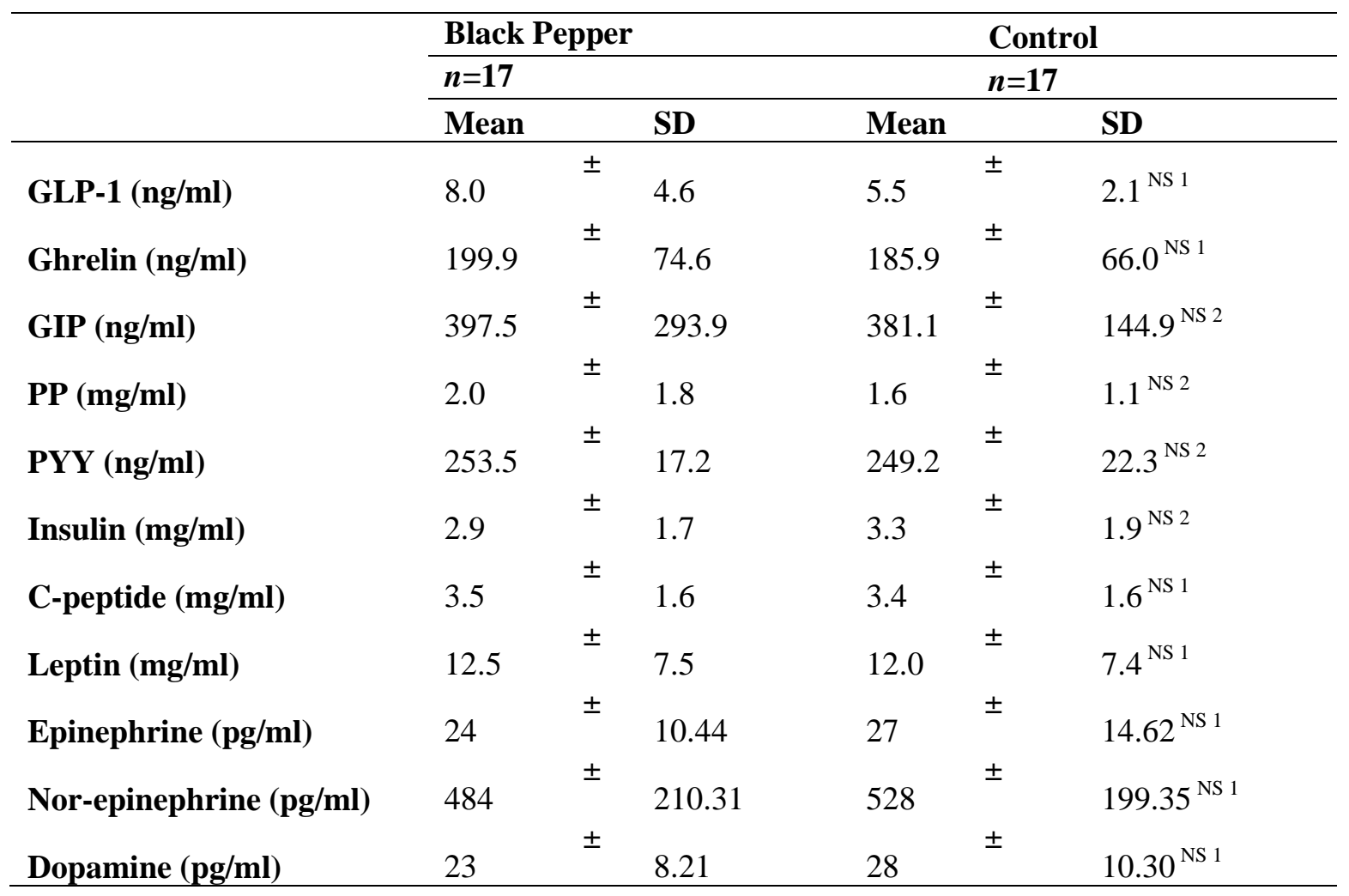

${ }^{1}$ Data displayed as mean \pm SD. ${ }^{\text {NS }}$ indicates not significantly different from no pepper control study day as assessed by paired t-tests ${ }^{1}$, or Wilcoxon Signed Rank Test ${ }^{2}$. GLP-1, glucagon-like peptide-1; GIP, gastric inhibitory polypeptide; PP, pancreatic polypeptide; PYY, peptide YY 


\section{DISCUSSION}

With the burden of obesity steadily rising, investigation of strategies to induce negative energy balance is essential. Ingredients which promote thermogenesis, fat oxidation, and satiety, without contributing kcal, may play a meaningful part in body weight management, as even small changes in energy balance $(\sim 0.21 \mathrm{MJ} /$ day $)$ may offset weight gain [17]. Several reports have suggested that piperine or other active components of BP, may enhance SNS activity and satiety [5, 18-19], however, contrary to our hypothesis, BP consumption did not alter EE, RQ, or plasma biomarkers of satiety.

In vitro and animal work suggests that active components of RP and BP impact thermogenesis, and that the mode of action (agonist at TRPV1) is similar in both cases. Considering this, parallels can be drawn between interventions examining the impact of RP on thermogenesis, substrate cycling and satiety, and the present study. Taken together, many of the most efficacious RP interventions have been conducted in Asian populations. In a series of studies in Japanese populations, 10g of RP resulted in a 30\% increase in EE [20-21]. However, the same investigators using 21.8g saw a substantially lower increase in EE (3.2\%) in Caucasians [22]. Gene-spice interactions may play a role in this. Similar to capsaicin, BP may be more efficacious in individuals harbouring single nucleotide polymorphisms (SNPs) in genes mediating the ingredient's effect. For example, a valine to leucine substitution in the TRPV1 receptor (TRPVI Val585Ile) is associated with increased abdominal fat loss responsiveness to capsinoids [23]. The allelic frequencies of several SNPs in TRPV1 which may modulate response are higher in Asian populations [24]. Even considering this, it is unclear whether BP consumption may alter EE, satiety or RQ in Asian populations, and if so, whether increased consumption would impact body weight.

The role of habitual consumption should be explored. In the case of RP, the decrease in $\mathrm{RQ}$ and rise in EE following acute consumption of capsaicin in some [20-21]. Japanese populations suggest that an acute effect is possible. Additional interventions in western Caucasian populations also induce some effect on RQ [4] and EE, suggesting that RP and capsaicin may also be efficacious in populations where consumption is not habitually high. Indeed, a recent study examining the impact of RP on EE and satiety suggests that an effect may be more pronounced in non-users [25]. How this applies to BP is unclear. As piperine induces greater tachyphylaxis and receptor desensitization than capsaicin at TRPV1 [5] the likelihood that repeated exposure would magnify the result is uncertain.

Piperine inhibits gastric emptying and transit time [26] which may delay the secretion of anorectic gut peptides, and hence delay their appearance in plasma. It is therefore possible that serial gut peptide measurement or visual analogue scales would have afforded a greater understanding of the satiating impact of BP. Inclusion of a single blood draw is a limitation of this study. BP was given orally as data suggests that sensory effects of capsaicin are important for satiety [27]. Considering the potential for sensory involvement, some earlier change in gut peptides may be expected at 30 minutes, even if a meal-induced peak was shifted to the right secondary to reduced transit time.

Previous studies with thermogenic ingredients have highlighted that satiating effects are modulated by energy intake status [28]. It is possible that the eucaloric study diet may have 
masked a potential benefit of $\mathrm{BP}$, and that a more pronounced impact may be observed with positive or negative energy balance.

This study supports the recent findings of Gregersen et al. who showed that acute consumption of BP did not alter DIT in young healthy men [29]. Due to the potential overshadowing effects of DIT, more subtle effects of BP may be revealed during the fasted or between-meal state, a time when functional ingredients would be most efficacious for weight management. This study builds substantially on the work of Gregersen et al, expanding our understanding of the effects of BP on other components of 24-hour EE. Additionally, this study was conducted in a metabolic chamber which provides more accurate assessment of subtle changes in EE no possible with a metabolic cart. Hence chamber studies may be required for definitive conclusions to be drawn.

\section{CONCLUSIONS}

Investigating the role of bioactive compounds is complex. Study design decisions should encompass population of choice, body composition [30] and energy balance. This study suggests that acute consumption of $1.5 \mathrm{~g}$ of $\mathrm{BP}$ is well tolerated and significantly increases circulating piperine. However, this dose does not influence 24-hour EE, RQ or postprandial biomarkers of satiety in post-menopausal, overweight Caucasian women under eucaloric conditions. Nevertheless, it is possible that BP administration in the between-meal or fasted state, a higher daily dose, or synergy arising from a combination with other thermogenic ingredients may have greater utility. Considering the potential benefits of thermogenic ingredients in the fight against obesity, continued research is warranted to determine populations who may maximally benefit from these dietary bioactives.

\section{ABBREVIATIONS}

BP, black pepper; EE, energy expenditure; RQ, respiratory quotient; NPC, no pepper control; RP, red pepper; TRPV1, human vanilloid receptor 1; SNS, sympathetic nervous system; USDA, United States Department of Agriculture; UNC NRI, University of North Carolina Chapel Hill Nutrition Research Institute; GIP, gastric inhibitory polypeptide; GLP-1, glucagon-like peptide 1; PYY, peptide tyrosine tyrosine; PP, pancreatic polypeptide; IRB, Institutional Review Board; $\mathrm{TSH}$, thyroid stimulating hormone; FFM, fat free mass; DXA, dual energy X-ray absorptiometry; RMR, Resting Metabolic Rate; PAL, physical activity level; SMR, sleeping metabolic rate; AEE, activity induced EE; UPLC-QTOFMS, ultra-performance liquid chromatography quadruple time-of-flight mass spectrometry

\section{Competing interests}

The authors declare no competing interests.

\section{Author contributions}

AGS, DCN and KDC designed the study. AOC conducted all aspects of the study and wrote the manuscript. All authors reviewed and approved the manuscript. 


\section{Author information}

None to declare

\section{Acknowledgements and funding}

We wish to thank Tondra Blevins, Jonni-Jo Carothers and Pamela Lambeth for their assistance and expertise throughout the study. This study was supported by the McCormick Science Institute.

\section{REFERENCES}

1. Hursel R, Westerterp-Plantenga MS. Thermogenic ingredients and body weight regulation. International journal of obesity 2013, 34:659-69.

2. Ludy MJ, Moore GE, Mattes RD. The effects of capsaicin and capsiate on energy balance: critical review and meta-analyses of studies in humans. Chemical senses 37 (2012) pp. 103-21.

3. Whiting S, Derbyshire E, Tiwari BK. Capsaicinoids and capsinoids. A potential role for weight management? A systematic review of the evidence. Appetite 2012, 59: 341-8.

4. Lejeune MP, Kovacs EM, Westerterp-Plantenga MS. Effect of capsaicin on substrate oxidation and weight maintenance after modest body-weight loss in human subjects. The British journal of nutrition 90 (2003) pp.651-59.

5. McNamara FN, Randall A, Gunthorpe MJ. Effects of piperine, the pungent component of black pepper, at the human vanilloid receptor (TRPV1). British journal of pharmacology 144 (2005) pp. 781-90.

6. Kawada T, Sakabe S, Watanabe T, Yamamoto M, Iwai K. Some pungent principles of spices cause the adrenal medulla to secrete catecholamine in anesthetized rats. Proceedings of the Society for Experimental Biology and Medicine Society for Experimental Biology and Medicine 1988, 188:229-33.

7. Bajad S, Bedi KL, Singla AK, Johri RK. Piperine inhibits gastric emptying and gastrointestinal transit in rats and mice. Planta medica 2001, 67:176-9.

8. Platel K Srinivasan K. Studies on the influence of dietary spices on food transit time in experimental rats. Nutrition Research, 2001, 21:1309-14.

9. Okumura Y, Narukawa M, Watanabe T. Adiposity suppression effect in mice due to black pepper and its main pungent component, piperine. Bioscience, biotechnology, and biochemistry 74 (2010a) pp. 1545-9.

10. USDA. Spice Supply and Disappearance, 1966-2005. Available at http://www.ers.usda.gov/Data/FoodConsumption.

11. Srinivasan K. Black pepper and its pungent principle-piperine: a review of diverse physiological effects. Critical reviews in food science and nutrition 2007, 47:735-48.

12. Srinivasan, K. Black pepper: overview of health benefits . Nutrition Today 2010, 45.

13. Arciero, PJ., Goran, MI., Poehlman, ET. Resting metabolic rate is lower in women than in men. Journal of applied physiology 1993 75:2514-20.

14. Brychta RJ, Rothney MP, Skarulis MC, Chen KY. Optimizing energy expenditure detection in human metabolic chambers. Annual International Conference of the IEEE 
Engineering in Medicine and Biology Society IEEE Engineering in Medicine and Biology Society Conference 2009, 6864-8.

15. Knab AM, Shanely RA, Corbin KD, Jin F, Sha W, Nieman DC. A 45-minute vigorous exercise bout increases metabolic rate for 14 hours. Medicine and science in sports and exercise 43 (2011) pp.1643-8.

16. Smeets AJ, Janssens PL, Westerterp-Plantenga MS. Addition of capsaicin and exchange of carbohydrate with protein counteract energy intake restriction effects on fullness and energy expenditure. The Journal of nutrition 2013, 143:442-7.

17. Hill JO, Wyatt HR, Reed GW, Peters JC. Obesity and the environment: where do we go from here? Science 2003, 299:853-5.

18. Okumura Y, Narukawa M, Iwasaki Y, Ishikawa A, Matsuda H, Yoshikawa M, et al. Activation of TRPV1 and TRPA1 by black pepper components. Bioscience, biotechnology, and biochemistry 2010b, 74:1068-72.

19. Eldershaw TP, Colquhoun EQ, Bennett KL, Dora KA, Clark MG. Resiniferatoxin and piperine: capsaicin-like stimulators of oxygen uptake in the perfused rat hindlimb. Life sciences 1994, 55:389-97.

20. Yoshioka M, Lim K, Kikuzato S, Kiyonaga A, Tanaka H, Shindo M, et al. Effects of red-pepper diet on the energy metabolism in men. Journal of nutritional science and vitaminology 1995, 41:647-56.

21. Yoshioka M, St-Pierre S, Suzuki M, Tremblay A. Effects of red pepper added to high-fat and high-carbohydrate meals on energy metabolism and substrate utilization in Japanese women. The British journal of nutrition 1998, 80:503-10.

22. Yoshioka M, Doucet E, Drapeau V, Dionne I, Tremblay A. Combined effects of red pepper and caffeine consumption on $24 \mathrm{~h}$ energy balance in subjects given free access to foods. The British journal of nutrition 2001, 85:203-11.

23. Snitker S, Fujishima Y, Shen H, Ott S, Pi-Sunyer X, Furuhata Y, et al. Effects of novel capsinoid treatment on fatness and energy metabolism in humans: possible pharmacogenetic implications. The American journal of clinical nutrition 2009, 89:4550.

24. Xu H, Tian W, Fu Y, Oyama TT, Anderson S, Cohen DM. Functional effects of nonsynonymous polymorphisms in the human TRPV1 gene. American journal of physiology renal physiology 2007, 293:F1865-76.

25. Ludy MJ, Mattes RD. The effects of hedonically acceptable red pepper doses on thermogenesis and appetite. Physiology \& behaviour 102 (2011) pp.251-8.

26. Badmaev V, Majeed M, Prakash L. Piperine derived from black pepper increases the plasma levels of coenzyme Q10 following oral supplementation. The journal of nutritional biochemistry. 2000, 11:109-13.

27. Westerterp-Plantenga MS, Smeets A, Lejeune MP. Sensory and gastrointestinal satiety effects of capsaicin on food intake. International journal of obesity 2005, 29:682-8.

28. Reinbach HC, Smeets A, Martinussen T, Moller P, Westerterp-Plantenga MS. Effects of capsaicin, green tea and $\mathrm{CH}-19$ sweet pepper on appetite and energy intake in humans in negative and positive energy balance. Clinical nutrition 2009, 28:260-5. 
29. Gregersen NT, Belza A, Jensen MG, Ritz C, Bitz C, Hels O et al. Acute effects of mustard, horseradish, black pepper and ginger on energy expenditure, appetite, ad libitum energy intake and energy balance in human subjects. The British journal of nutrition 2012, 5:1-8.

30. Yoneshiro T, Aita S, Kawai Y, Iwanaga T, Saito M. Nonpungent capsaicin analogs (capsinoids) increase energy expenditure through the activation of brown adipose tissue in humans. The American journal of clinical nutrition 2012, 95:845-50. 